\title{
Silviculture as a tool to support stability and diversity of forests under climate change: study from Krkonoše Mountains
}

\author{
Zdeněk Vacek ${ }^{1 *}$, Anna Prokůpková ${ }^{1}$, Stanislav Vacek ${ }^{1}$, Jan Cukor ${ }^{1,2}$, Lukáš Bílek ${ }^{1}$, \\ Josef Gallo ${ }^{1}$, Daniel Bulušek ${ }^{1}$ \\ ${ }^{1}$ Czech University of Life Sciences Prague, Faculty of Forestry and Wood Sciences, Kamýcká 129, CZ - 16500 Prague - Suchdol, \\ Czech Republic \\ ${ }^{2}$ Forestry and Game Management Research Institute, Strnady 136, CZ - 25202 Jíloviště, Czech Republic
}

\begin{abstract}
In Europe, warming, droughts and the rise of extreme climate events have an increasing significant negative effect on forest stands. Therefore, it is necessary to create appropriate adaptation strategies of silviculture to mitigate the impacts of global climate change on forest ecosystems in Central Europe. The objectives of this paper were to evaluate stand production, structure and diversity on eight experimental research plots in the Krkonoše Mountains. Subsequently, three variants of management were compared in mixed stands at the age of $17-20$ years originating from natural regeneration: A) control variant (stands before thinning), B) applied newly designed thinning in the context of climate change adaptation and C) simulative thinning from below. Number of trees decreased from 3,256 trees ha ${ }^{-1}$ by $32 \%$ after adaptation thinning and by $36 \%$ after thinning from below. The basal area decreased in variant B by $22 \%$ and in variant $C$ by $12 \%$. Structural diversity and tree species richness increased after application of adaptation thinning, while decrease of diameter differentiation and total diversity was observed after thinning from below. Moreover, horizontal structure changed from aggregated spatial pattern to random distribution after the interventions, especially under adaptation thinning. The newly designed structuralizing adaptation thinning method seems to be a more suitable option in given habitat and stand conditions compared to the commonly performed thinning from below. In future, this issue will certainly require further close cooperation of forestry experts in order to arrive at optimal variants of solutions differentiated according to specific conditions.
\end{abstract}

Key words: thinning; forest adaptation; stand structure; timber production; Central Europe

Editor: Igor Štefančík

\section{Introduction}

Climate change substantially affects the geographical distribution of plant species worldwide (Bolte et al. 2010; Rigling et al. 2013; Scheffers et al. 2016). Most of the changes are associated with an increase in air temperature, decreasing rainfall during the growing season, and extreme climate events such as prolonged droughts, more frequent storms or uneven occurrence of precipitation (Allen et al. 2010; IPCC 2013; Spathelf et al. 2014). Global warming also increases the incidence of insect outbreaks, fungal pathogens and forest fires (Jönsson et al. 2009; Seidl et al. 2014; Bošel'a et al. 2018). These unfavourable conditions induce changes in tree species such as acclimatization and phenotypic plasticity, local adaptation, migration and mortality (Bussotti et al. 2015). For these reasons, forest ecosystem management deserves an increased attention (Knoke et al. 2008; Seidl et al. 2016; Dyderski et al. 2017).
The adaptation of forests to climate change is both a major challenge and a long-term task for European forestry (Lindner et al. 2010). Strategic decision-making in forest policy in Europe will have to rely on objective scientific knowledge with some uncertainty at local, regional and continental levels (Lindner et al. 2014; O'Connor et al. 2015). However, knowledge about the response of forest ecosystems to climate change is still insufficient or only partial (Pretzsch et al. 2014; Gazol et al. 2015). Forests of Central and Northern Europe are known to have increased their growth in the last century (Pretzsch et al. 2014). In contrast, many tree species suffer from drought stress at the southern edge of their current distribution in Europe and are likely to shift their span to the north and to higher altitudes (Rabasa et al. 2013; Saltré et al. 2014; Kozak \& Parpan 2019). According to Hanewinkel et al. (2013), Norway spruce (Picea abies [L.] Karts.), the most commercial tree species in Europe, will only find 
appropriate conditions for growth in Central Europe at the highest elevations by the end of this century. Deciduous stands (beech and oak) should be dominant in Western, Central and Eastern Europe (Štefančík et al. 2018). Mainly Mediterranean oak species should be utilized in southern Europe (Moreno-Fernández et al. 2016). European beech (Fagus sylvatica L.), as ecologically and economically one of the most important native tree species in Europe, has experienced varied changes across the continent depending on the site quality and the altitudinal conditions of the environment (Piovesan et al. 2008; Aertsen et al. 2014; Podrázský et al. 2019). The situation was similar in the Czech Republic (Vinš et al. 1997) and in the study area of Krkonoše Mts., where it was originally an important dominant tree species (Vacek \& Hejcman 2012; Bulušek et al. 2016; Šimůnek et al. 2019). Bošela et al. (2016) report from the Carpathians in this respect that the increase of temperature in the growing season together with sufficient rainfall had a positive effect on the growth of beech. In contrast, Zimmermann et al. (2015) in Central Europe indicate the increasing temperature and intensity of droughts during the growing season as the main limiting factors for beech and warn against its cultivation in the area of interest.

The search for appropriate adaptive management practices in specific natural conditions should be based on structural and developmental knowledge of local natural forests in recent decades (Hemberet al. 2012; Bošel'a et al. 2016; Vacek et al. 2019b). It will ordinarily be a set of cultivation measures (especially thinning and regeneration cutting) known from the past, which must be tested in changing specific habitats and stand conditions. O'Hara (2015) states that forestry should consider a number of new facts and opportunities when seeking ways of adaptive management, especially from the point of view of climate, invasive organisms, pests and introduced tree species. In particular, the continuous cover forests absorb climate extremes most effectively, improves carbon sequestration and effectively manage water (Pokorný 2013). It is however a question what species, age, spatial and genetic structure should we seek for to secure the permanence of quantity, quality and safety of production.

For ecological stability and production safety, forests should be differentiated in terms of species, spatial and age structure (Brang et al. 2014). In case of quality and quantity of timber production, it will depend mainly on the composition and structure of the forest (Coomes et al. 2014; Vacek et al. 2014), which are highly dependent on management methods (Vacek et al. 2015a). In mountain locations, forests can be managed by shelterwood silvicultural system, but it can also be a selection or partial cutting method or combination of both (Gallo et al. 2020). At the same time, highly important question is how to optimize the tending of existing stands (Štefančík 2015). Bošel'a et al. (2016) showed that the ways of tending the beech stands significantly influence not only the quality and quantity of production, but also their vitality. Beech trees in the lower stand layers have not responded to an increase in temperature, suggesting that vertically diversified forests could be more stable under the conditions of warming climate. Primicia et al. (2015), on the other hand, states that trees with a high level of competition, especially in the lower layers, may be very sensitive to drought-heat stress in the future, as beech is known to be a sensitive species to temperature extremes in the initial stage of growth (Gallo et al. 2017).

Adequate species composition and form of mixing also play an important role in forest management under the unfavorable conditions of changing climate (Neuner et al. 2013; Vitali et al. 2018; Vacek et al. 2019a). Mixed forests are much more effective at mitigating the negative impacts of changing environmental conditions, especially in the cases of growth and survival (Albert et al. 2015; Pukkala 2018) and can even be more productive under climate change (Podrázský et al. 2014; Liang et al. 2016). In addition, the selection of a suitable species composition has a significant effect on carbon sequestration (Andivia et al. 2016; Cukor et al. 2017). In this respect, mixed forests have been more frequently established in Europe over the last two decades (Bravo-Oviedo et al. 2014; Forrester \& Pretzsch 2015; del Río et al. 2016; Metz et al. 2016; Pretzsch \& Biber 2016) and it is necessary to obtain exact knowledge about their management (Fahlvik et al. 2005; Agestam et al. 2006; Hynynen et al. 2011; Novák et al. 2017). Knowledge of thinning in various stand mixtures is not yet sufficient (Štefančík \& Kamenský 2006; Štefančík 2010; Prévost \& Gauthier 2012; Dhar et al. 2015; Drössler et al. 2015), especially in terms of stand growth, stability and vitality. Based on the described changes, the forest management practices should be significantly changed in following decades.

The aims of the presented study conducted in mixed forest stands (age of $17-20$ years) in the western Krkonoše Mountains were to: (1) design a new optimized structuralizing thinning intervention in support of stand stability and diversity in the context of climate change adaptation, (2) determine basic stand characteristics, timber volume, structure (diameter, height) and diversity (species, structural, total) of mixed stands in the study area and (3) compare the state of the forest stands A) before the tending intervention, B) immediately after the model application of the newly designed structuralizing thinning intervention and $\mathrm{C}$ ) after the simulative performance of the thinning from below.

\section{Material and methods}

\subsection{Study area}

The research was conducted in the western part of the Krkonoše Mountains close to the village Končiny, north part of the Czech Republic (GPS: $50^{\circ} 42^{\prime} 45^{\prime \prime} \mathrm{N}$,

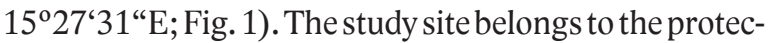


tion zone of Krkonoše Mountains National Park at elevation of $581-597 \mathrm{~m}$ a.s.l. and slope of $12-19^{\circ}$ with east to north-eastern exposure. Annual temperature of the locality is $7.2^{\circ} \mathrm{C}$, and the annual precipitation varies around $940 \mathrm{~mm}$. The highest amount of precipitation is in July (113 mm), the lowest in October (52 mm; Fig. 1). The warmest month is in July $\left(16.5^{\circ} \mathrm{C}\right)$, the coldest month in January $\left(-2.2^{\circ} \mathrm{C}\right)$. The growing season lasts about 130 days $\left(\mathrm{T}_{\max } \geq 10^{\circ} \mathrm{C}\right)$ with an average temperature of $13.1^{\circ} \mathrm{C}$ and a total precipitation of $530 \mathrm{~mm}$. The average number of days with snow cover is 135 days and the number of days with precipitation $\left(\mathrm{P}_{\max } \geq 1 \mathrm{~mm}\right)$ is 125 days. The study area is qualified as a humid continental climate zone, characterized by warm to hot, humid summers and cold to severely cold winters (Dfb) according to the Köppen climate classification (Köppen 1931). According to the detailed Quitt climate classification (Quitt 1971), the area can be classified in the cold climatic region (subregion $\mathrm{CH}$ 6). The parent rock consists mainly of slate, phyllites and schist. Modal cambisol is the predominant soil type.

In the area, 8 experimental research plots (ERP) in two forest stands (4 ERP in each stand) were established. Both forest stands originated from natural regeneration. The first studied stand (ERP 1 - 4) was composed of European beech (Fagus sylvatica L.), Sycamore maple (Acerpseudoplatanus L.), European ash (Fraxinus excelsior L.) and rowan (Sorbus aucuparia L.) of mean age 20 years. Second studied stand (ERP 5 - 8) was composed of main tree layer of silver birch (Betula pendula Roth.), rowan and beech of mean age 17 years under the standards of beech and silver fir (Abies alba Mill.) of mean age 51 years. In both stands Norway spruce (Picea abies
[L.] Karst.), European aspen (Popolus tremula L.), wild cherry (Prunus avium L.) and goat willow (Salix caprea L.) were admixed tree species. Ash trees were weakened by Hymenoscyphus fraxineus (T. Kowalski) and attacks by Hylesinus fraxini (Panzer). In terms of phytocoenology, all ERP belonged to Abieto-Fagetum acidophilum (5K - Acidic Fir-Beech; Viewegh et al. 2003) and the alliance Luzulo-Fagion sylvaticae Lohmeyer et Tüxen in Tüxen 1954.

\subsection{Data collection}

Field-Map technology (IFER-Monitoring and Mapping Solutions Ltd.) was used to determine the structure of the tree layer of mixed forest stands on 8 ERP of $12.5 \times$ $12.5 \mathrm{~m}$ in 2019 . The positions of all trees with diameter at breast height $(\mathrm{DBH}) \geq 4 \mathrm{~cm}$ were localized. The height of the live crown base and the crown projection were also measured in the tree layer, at least in 4 directions perpendicular to each other. Diameters of the tree layer were measured by a Mantax Blue metal calliper (Haglöf, Sweden) with an accuracy of $1 \mathrm{~mm}$ and heights were measured using a Vertex laser hypsometer (Haglöf, Sweden) with an accuracy of $0.1 \mathrm{~m}$. According to the classification by Kraft (1884), the social position of the trees within a stand was assessed: 1 - predominant, 2 - dominant, 3 co-dominant, 4 - sub-dominant, 5 - suppressed.

Subsequently, two thinning interventions were marked (trees selected for harvesting) in the stands on all the 8ERP: (B) newly designed climate change adaptation thinning (hereinafter "adaptation thinning”) described in Table 1 and (C) thinning from below (harvested subdominant and suppressed trees) according to Kraft clas-

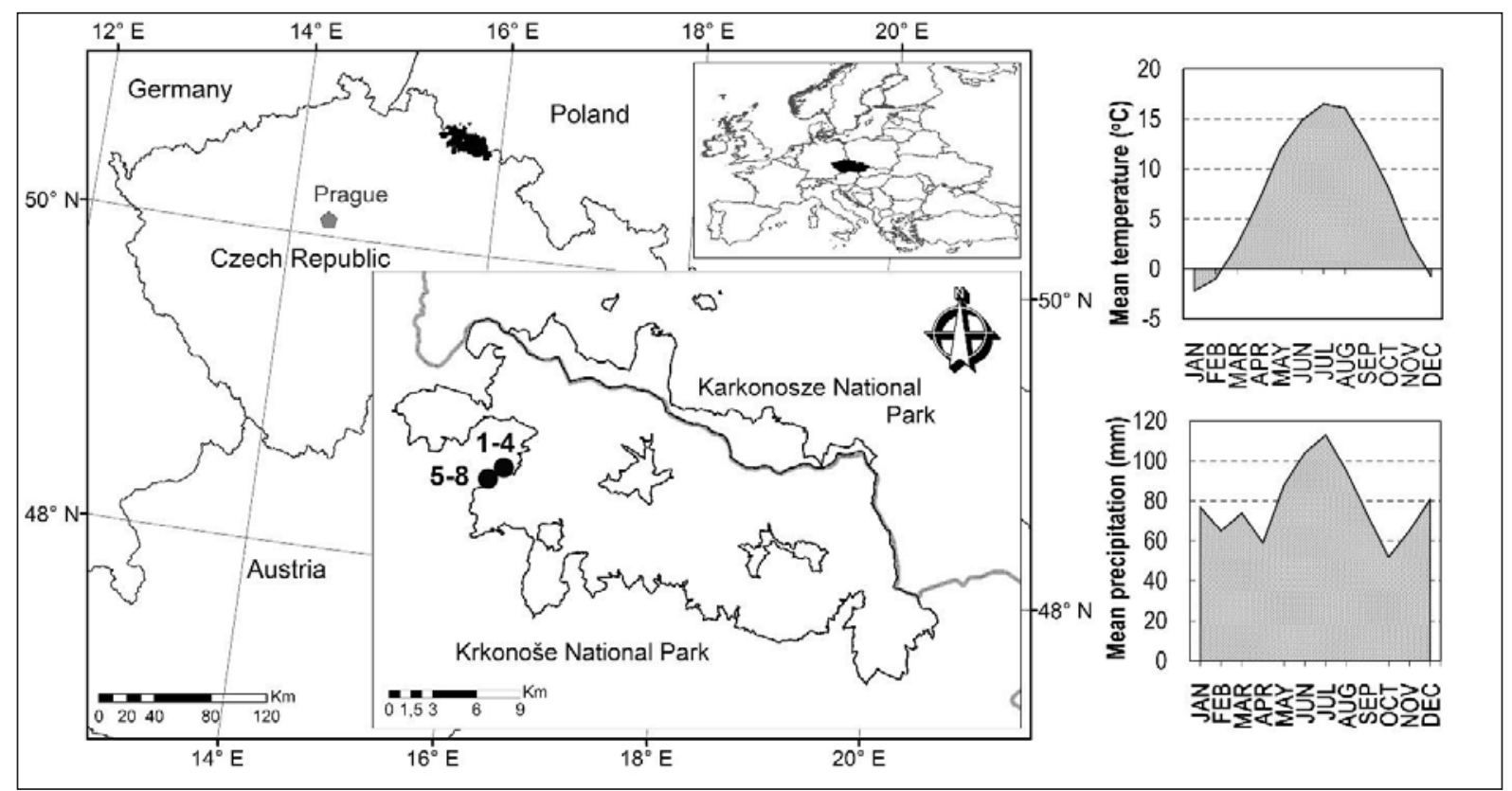

Fig. 1. Localization of mixed stands on experimental research plots Končiny 1 - 8 in Krkonoše Mountains National Park (protection zone) and mean monthly climatic values (1960 - 2018); map was made in software ArcGIS 10 (Esri). 
sification with the support of natural tree species composition (preferentially spruce and beech at the expense of other tree species), which is applied in classical forest management on study site (Fig. 2); both tending methods were compared with the stands before the intervention (A). This new structuralizing adaptation thinning supporting admixed tree species was created in cooperation with the employees of Department of Silviculture, Faculty of Forestry and Wood Sciences, Czech University of Life Sciences in Prague, Czech Forestry Society (the largest forestry professional association in the Czech Republic), Forestry and Game Management Research Institute, Colloredo-Mannsfeld spol. s.r.o. (the largest private forest owner in the Czech Republic) and Jilemnice municipality forests. A form with 10 indicators was cre- ated by main author according to references (Slodičák \& Novák 2007; Cordonnier et al. 2013; Pokorný 2013; Forest Europe 2015; Vacek et al. 2019a) and subsequently the experts in silvicultural field (15 academic and practical persons) numbered the indicators according to the importance from 1 (min.) - 10 (max.) in relation to mitigate climate change. In order to simplify this thinning variant, the 3 least rated indicators were excluded from the evaluation. Trees selected for harvesting were documented in Field-Map technology. From January to February 2020, adaptation thinning was applied on all 8 ERP and immediate stand changes were compared with the simulative thinning from below.

Table 1. Considered suitable objectives with description and relevance of the newly designed climate change adaptation thinning.

\begin{tabular}{lll}
\hline Order of importance & \multicolumn{1}{c}{ Indicators of thinning choice } & \multicolumn{1}{c}{ Description } \\
\hline 1 & Species composition addressing climate change & $\begin{array}{l}\text { adaptable tree species (for drought, climate extremes), shift of forest vegetation zones, } \\
\text { optimal stand mixtures in terms of ecological criteria (valency, susceptibility to damage, } \\
\text { tolerance and adaptability of tree species) } \\
\text { resistance, vitality, foliation, abiotic and biotic damage } \\
\text { species richness (number of tree species), heterogeneity and evenness } \\
\text { diameter and height (age) differentiation, vertical canopy, horizontal structure }\end{array}$ \\
3 & Health status & silvicultural potential, stem quality (straightness, defects), crown quality (length, regularity, \\
4 & Species diversity & deflection) \\
5 & Structural diversity & timber, growth tendencies; periodic, mean and value increment \\
6 & Quality of production & canopy conservation, area and distribution of crown projections
\end{tabular}

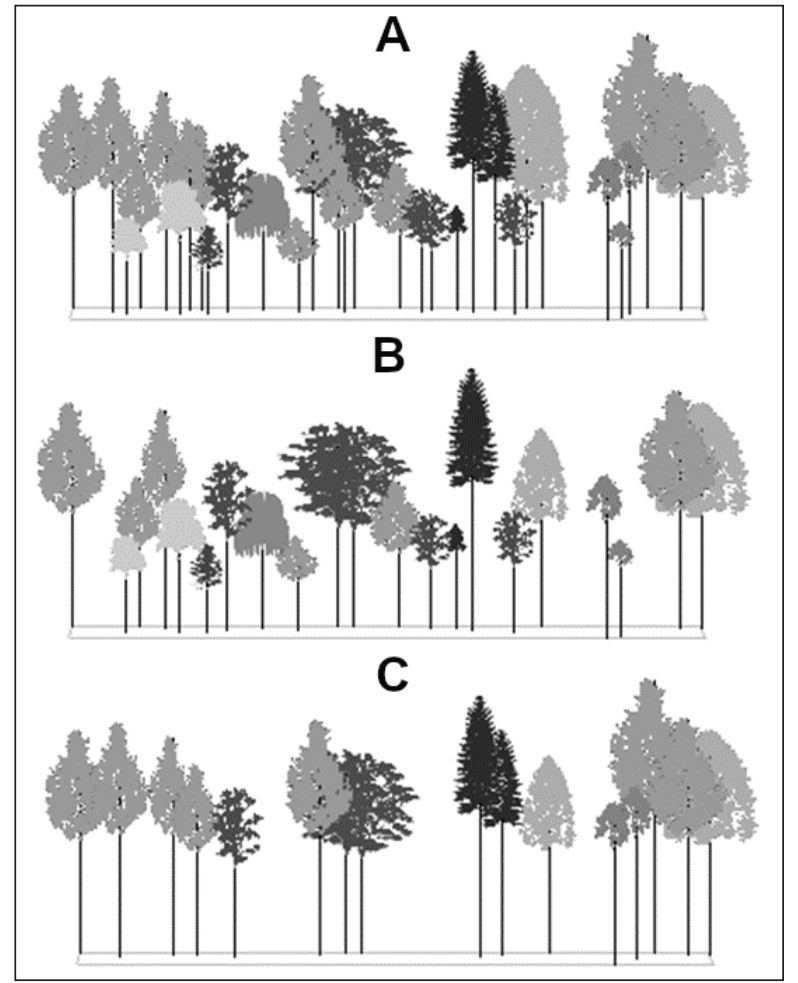

Fig. 2. Vertical schema of mixed forest stand A) before thinning, B) after application of the climate change adaptation thinning and $\mathrm{C}$ ) thinning from below; figure was made in software SIBYLA Triquetra 10 (Fabrika \& Pretzsch, TUZVO).

\subsection{Data analysis}

The basic structure, diversity and production characteristics of the tree layer were evaluated by the SIBYLA Triquetra 10 forest growth simulator (Fabrika \& Durský 2005). The PointPro 2.1 (Zahradník \& Puš, ČZU) program was used to calculate the characteristics of the horizontal layout of the individuals on the plots. For the evaluation of the spatial pattern, the following indicators were computed: index of non-randomness (Pielou 1959; Mountford 1961), aggregation index (Clark \& Evans 1954), index of cluster size (David \& Moore 1954) based on the 10 quadrats and Ripley $L$-function (Ripley 1981). The test of the significance of deviations from the values expected for the random arrangement of the points was made using Monte Carlo simulations. The mean values of $L$-function were estimated as arithmetic means from $L$-functions computed for 999 randomly generated point structures. Species and structural diversity were evaluated by species richness (Margalef 1958), species heterogeneity (Shannon 1948), species evenness (Pielou 1975), Arten-profile index (Pretzsch 2006), diameter and height differentiation (Füldner 1995), crown differentiation and total stand diversity (Jaehne \& Dohrenbusch 1997; Table 2). Stand volume was calculated according to Petráš \& Pajtík (1991). The relative stand density index (SDI; Reineke 1933) and the crown closure (CC; Crookston $\&$ Stage 1999) were observed for each plot. 
Table 2. Overview of indices describing the stand diversity and their common interpretation.

\begin{tabular}{|c|c|c|c|c|}
\hline Criterion & Quantifiers & Label & Reference & Evaluation \\
\hline & Richness & $D(\mathrm{Mi})$ & Margalef (1958) & minimum $D=0$, higher $D=$ higher values \\
\hline \multirow[t]{2}{*}{ Species diversity } & Heterogeneity & $H^{\prime}(\mathrm{Si})$ & Shannon (1948) & minimum $H^{\prime}=0$, higher $H^{\prime}=$ higher values \\
\hline & Evenness & $E(\mathrm{Pii})$ & Pielou (1975) & range $0-1 ;$ minimum $E=0$, maximum $E=1$ \\
\hline \multirow[t]{3}{*}{ Horizontal structure } & $\begin{array}{l}\text { Index } \\
\text { of non-randomness }\end{array}$ & $\alpha(\mathrm{P} \& \mathrm{Mi})$ & $\begin{array}{l}\text { Pielou (1959); } \\
\text { Mountford (1961) }\end{array}$ & mean value $\alpha=1$; aggregation $\alpha>1$; regularity $\alpha<1$ \\
\hline & Aggregation index & $R(\mathrm{C} \& \mathrm{Ei})$ & Clark \& Evans (1954) & mean value $R=1$; aggregation $R<1$; regularity $R>1$ \\
\hline & Index of cluster size & $C S(\mathrm{D} \& \mathrm{Mi})$ & David \& Moore (1954) & mean value $C S=0 ;$ aggregation $C S>0 ;$ regularity $C S<0$ \\
\hline Vertical structure & Arten-profile index & $A($ Pri) & Pretzsch (2006) & range $0-1$; balanced vertical structure $A<0.3$; selection forest $A>0.9$ \\
\hline Structure differentiation & $\begin{array}{l}\text { Diameter dif. } \\
\text { Height dif. }\end{array}$ & $\begin{array}{l}T M_{d}(\mathrm{Fi}) \\
T M_{b}(\mathrm{Fi})\end{array}$ & Füldner (1995) & range $0-1$; low $T M<0.3$; very high differentiation $T M>0.7$ \\
\hline Complex diversity & Stand diversity & $B(\mathrm{~J} \& \mathrm{Di})$ & Jaehne \& Dohrenbusch (1997) & $\begin{array}{c}\text { monotonous structure } B<4 \text {; uneven structure } B=6-8 \text {; } \\
\text { very diverse structure } B>9\end{array}$ \\
\hline
\end{tabular}

Unconstrained principal component analysis (PCA) in Canoco 5 (Šmilauer \& Lepš 2014) was used to analyze the relationships between stand structure, timber production and diversity of three variants of management. Data were log-transformed, centred and standardized before the analysis. The results of PCA were exported into the form of ordination diagram.

\section{Results}

\subsection{Stand characteristics, structure and timber volume}

Tree density ranged from 2,112 to 5,184 trees per ha ${ }^{-1}$ before thinning (control variant A), after model adaptation thinning (variant $\mathrm{B}$ ) number of trees was between 1,280 to 3,968 trees $\mathrm{ha}^{-1}$ and after simulative thinning frombelow (variantC) itwas from 1,408 to 3,008 trees ha $^{-1}$ (Table 3). Number of trees decreased from mean density of 3,256 trees ha-1 by $32 \%$ after adaptation thinning (to 2,192 trees $\mathrm{ha}^{-1}$ ) and by $36 \%$ after thinning from below (to 2,096 trees $\mathrm{ha}^{-1}$ ). In variant $\mathrm{A}$, basal area ranged between $21.8-39.2 \mathrm{~m}^{2} \mathrm{ha}^{-1}$ and stand volume reached $127-377 \mathrm{~m}^{3} \mathrm{ha}^{-1}$. After thinning interventions, stand volume decreased from 185 to $159 \mathrm{~m}^{3} \mathrm{ha}^{-1}$ in variant B (-14\%) and to $175 \mathrm{~m}^{3} \mathrm{ha}^{-1}$ in variant C (-6\%). The basal area decreased in variant $\mathrm{B}$ by $22 \%$ and in variant $\mathrm{C}$ by $12 \%$. On the other hand, mean DBH increased by $8 \%$ after adaptation thinning, respectively by $16 \%$ after thinning from below. Similar positive trend after interventions was observed in stem volume and stand height. Mean height was higher in variant $C(12.5 \mathrm{~m})$ compared to variant $A$ $(10.8 \mathrm{~m})$ and variant $B(11.2 \mathrm{~m})$. SDI decreased from 0.75 to 0.58 in variant $B$ and to 0.63 in variant $C$. The highest differences among thinning variants were observed in tree density and mean height.

The diameter frequency on ERP 1-8 before thinning (variant A) showed visible left-skewed selection distribution, where beech dominated the overstorey and birch, beech and rowan mostly formed the understory (Fig. 3 and 4). Sycamore was evenly represented across diameter classes. Rowan was observed only in diameter to $12 \mathrm{~cm}$. Birch was dominant in all diameter classes to $20 \mathrm{~cm}$. After adaptation thinning number of trees decreased in classes

Table 3. Basic stand characteristics on experimental research plots 1- 8 in A) control variant (before thinning), B) after applied climate change adaptation thinning and $\mathrm{C}$ ) after simulative thinning from below.

\begin{tabular}{|c|c|c|c|c|c|c|c|c|c|c|c|c|c|c|c|c|c|c|c|c|c|}
\hline ERP & Type & $\begin{array}{c}\mathrm{dbh} \\
{[\mathrm{cm}]}\end{array}$ & & $\begin{array}{c}\mathrm{h} \\
{[\mathrm{m}]}\end{array}$ & & $\begin{array}{c}\mathrm{v} \\
{\left[\mathrm{m}^{3}\right]}\end{array}$ & & f & & $\begin{array}{c}\mathrm{N} \\
\text { trees ha }^{-}\end{array}$ & & $\begin{array}{c}\text { BA } \\
{\left[\mathrm{m}^{2} \mathrm{ha}^{-1}\right.}\end{array}$ & & $\begin{array}{c}\mathrm{V} \\
{\left[\mathrm{m}^{3} \mathrm{ha}^{-}\right.}\end{array}$ & & HDR & & $\begin{array}{r}\text { CC } \\
{[\%]}\end{array}$ & & $\mathrm{SD}$ & \\
\hline & $\mathrm{A}$ & 11.9 & & 10.3 & & 0.069 & & 0.596 & & 3,520 & & 39.2 & & 242 & & 87.3 & & 99.2 & & 0.91 & \\
\hline \multirow[t]{3}{*}{1} & B & 13.8 & $\nearrow$ & 11.4 & $\nearrow$ & 0.100 & $\nearrow$ & 0.584 & $\nearrow$ & 2,112 & 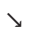 & 31.4 & $\searrow$ & 212 & $\searrow$ & 83.1 & $\searrow$ & 99.2 & $\searrow$ & 0.68 & $\searrow$ \\
\hline & $\mathrm{C}$ & 14.0 & $\lambda$ & 12.2 & $\lambda$ & 0.099 & $\pi$ & 0.527 & $\nearrow$ & 2,432 & $\searrow$ & 37.2 & $\searrow$ & 241 & $\searrow$ & 87.4 & $\lambda$ & 98.7 & $\searrow$ & 0.82 & $\searrow$ \\
\hline & $\mathrm{A}$ & 10.0 & & 9.4 & & 0.038 & & 0.519 & & 3,712 & & 29.2 & & 142 & & 94.1 & & 99.4 & & 0.77 & \\
\hline \multirow[t]{3}{*}{2} & B & 11.0 & $\nearrow$ & 9.5 & $\nearrow$ & 0.051 & $\nearrow$ & 0.563 & $\nearrow$ & 2,304 & $\searrow$ & 21.7 & $\searrow$ & 118 & $\searrow$ & 86.8 & $\searrow$ & 98.6 & $\searrow$ & 0.55 & $\searrow$ \\
\hline & C & 11.9 & $\lambda$ & 10.8 & $\lambda$ & 0.058 & $\nearrow$ & 0.482 & $\lambda$ & 1,920 & $\searrow$ & 21.1 & $\searrow$ & 112 & $\searrow$ & 91.4 & $\searrow$ & 98.1 & $\searrow$ & 0.53 & $\searrow$ \\
\hline & $\mathrm{A}$ & 9.1 & & 10.0 & & 0.031 & & 0.475 & & 4,096 & & 26.4 & & 127 & & 110.3 & & 99.4 & & 0.75 & \\
\hline \multirow[t]{3}{*}{3} & B & 9.3 & $\nearrow$ & 10.0 & $\searrow$ & 0.035 & 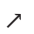 & 0.509 & $\pi$ & 2,752 & $\searrow$ & 18.5 & $\searrow$ & 95 & $\searrow$ & 107.6 & y & 98.9 & $\searrow$ & 0.52 & $\searrow$ \\
\hline & $\mathrm{C}$ & 10.4 & $\lambda$ & 11.4 & $\lambda$ & 0.044 & $\pi$ & 0.452 & $\lambda$ & 2,880 & $\searrow$ & 24.3 & $\searrow$ & 126 & $\searrow$ & 110.0 & $\searrow$ & 99.1 & $\searrow$ & 0.66 & $\searrow$ \\
\hline & $\mathrm{A}$ & 11.7 & & 10.8 & & 0.070 & & 0.601 & & 2,368 & & 25.2 & & 166 & & 92.5 & & 99.2 & & 0.65 & \\
\hline \multirow[t]{3}{*}{4} & B & 11.1 & $\searrow$ & 10.5 & $\searrow$ & 0.065 & $\searrow$ & 0.634 & $\searrow$ & 1,728 & $\searrow$ & 16.4 & $\searrow$ & 112 & $\searrow$ & 94.9 & $\searrow$ & 97.4 & $\searrow$ & 0.45 & $\searrow$ \\
\hline & $\mathrm{C}$ & 13.4 & $\nearrow$ & 12.3 & $\lambda$ & 0.097 & $\lambda$ & 0.557 & $\nearrow$ & 1,664 & $\searrow$ & 23.3 & $\searrow$ & 162 & $\searrow$ & 92.2 & $\searrow$ & 98.7 & $\searrow$ & 0.55 & $\searrow$ \\
\hline & A & 12.5 & & 12.0 & & 0.067 & & 0.455 & & 2,624 & & 31.8 & & 177 & & 96.4 & & 99.4 & & 0.77 & \\
\hline \multirow[t]{3}{*}{5} & B & 13.9 & $\nearrow$ & 12.8 & $\nearrow$ & 0.091 & $\nearrow$ & 0.469 & $\nearrow$ & 1,664 & $\searrow$ & 25.1 & $\searrow$ & 152 & $\searrow$ & 92.2 & $\searrow$ & 98.9 & $\searrow$ & 0.58 & $\searrow$ \\
\hline & $\mathrm{C}$ & 14.0 & $\nearrow$ & 13.5 & $\lambda$ & 0.091 & $\pi$ & 0.435 & $\nearrow$ & 1,792 & $\searrow$ & 27.4 & $\searrow$ & 163 & $\searrow$ & 96.6 & $\nearrow$ & 99.2 & $\searrow$ & 0.63 & $\searrow$ \\
\hline & $\mathrm{A}$ & 8.4 & & 11.4 & & 0.021 & & 0.332 & & 5,184 & & 28.7 & & 109 & & 136.3 & & 99.5 & & 0.85 & \\
\hline \multirow[t]{3}{*}{6} & B & 8.7 & $\nearrow$ & 11.7 & $\nearrow$ & 0.023 & $\nearrow$ & 0.336 & $\nearrow$ & 3,968 & $\searrow$ & 23.6 & $\searrow$ & 93 & $\searrow$ & 134.5 & $\searrow$ & 99.4 & $\searrow$ & 0.69 & $\searrow$ \\
\hline & $\mathrm{C}$ & 9.5 & $\nearrow$ & 12.9 & $\lambda$ & 0.029 & $\nearrow$ & 0.312 & $\nearrow$ & 3,008 & $\searrow$ & 21.2 & $\searrow$ & 86 & $\searrow$ & 136.5 & $\nearrow$ & 99.2 & $\searrow$ & 0.61 & $\searrow$ \\
\hline & $\mathrm{A}$ & 14.1 & & 11.5 & & 0.155 & & 0.861 & & 2,432 & & 37.8 & & 377 & & 81.8 & & 99.2 & & 0.78 & \\
\hline \multirow[t]{3}{*}{7} & B & 16.3 & $\nearrow$ & 12.4 & $r$ & 0.215 & $\pi$ & 0.829 & $\nearrow$ & 1,728 & y & 35.3 & $\searrow$ & 372 & $\searrow$ & 76.3 & y & 98.5 & $\searrow$ & 0.70 & $\searrow$ \\
\hline & $\mathrm{C}$ & 16.8 & $\lambda$ & 13.5 & $\lambda$ & 0.226 & $\nearrow$ & 0.755 & $\nearrow$ & 1,664 & $\searrow$ & 36.5 & $\searrow$ & 376 & 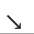 & 80.4 & $\searrow$ & 98.7 & 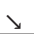 & 0.73 & $\searrow$ \\
\hline & $\mathrm{A}$ & 11.5 & & 11.1 & & 0.067 & & 0.575 & & 2,112 & & 21.8 & & 141 & & 96.9 & & 98.7 & & 0.52 & \\
\hline \multirow[t]{2}{*}{8} & B & 12.8 & $\nearrow$ & 11.6 & $\nearrow$ & 0.091 & $\nearrow$ & 0.610 & $\nearrow$ & 1,280 & $\searrow$ & 16.1 & $\searrow$ & 117 & $\searrow$ & 91.1 & $\searrow$ & 95.2 & $\searrow$ & 0.41 & $\searrow$ \\
\hline & $\mathrm{C}$ & 13.7 & $\nearrow$ & 13.2 & $\lambda$ & 0.099 & $\pi$ & 0.508 & $\nearrow$ & 1,408 & $\searrow$ & 20.5 & 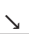 & 139 & 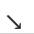 & 96.5 & $\searrow$ & 97.7 & $\searrow$ & 0.49 & $\searrow$ \\
\hline
\end{tabular}

Notes: $\mathrm{dbh}$ - mean quadratic diameter at breast height, $\mathrm{h}$ - mean height, $\mathrm{v}$ - mean tree volume, $\mathrm{f}$ - form factor, $\mathrm{N}$ - number of trees per hectare, $\mathrm{BA}$ - basal area, $\mathrm{V}$ - stand volume, $\mathrm{HDR}$ - height to diameter ratio, CC - canopy closure, SDI - stand density index; changes: $\downarrow$ - decrease, $>$ - increase. 
from 4 to $16 \mathrm{~cm}$, while the center of thinning interest for variant $C$ ranged from 4 to $8 \mathrm{~cm}$. After thinning, the highest decrease in tree numbers was observed in first class $4-6 \mathrm{~cm}$ in both variants - in variant B by -448 trees $\mathrm{ha}^{-1}(-34 \%)$ and especially in variant $\mathrm{C}$ by -960 trees ha $^{-1}$ $(-74 \%)$. In variant $\mathrm{B}$, the percentage of harvested trees was evenly distributed across diameter classes, while for variant $C$ the percentage of harvested trees rapidly decreased with increasing diameter. In variant $\mathrm{B}$, the most frequent diameter class have been still $4-6 \mathrm{~cm}$, while it was moved to class $6-8 \mathrm{~cm}$ in variant $C$.

\subsection{Biodiversity of tree layer}

Before thinning (variantA), the main tree species on ERP 1 - 8 was birch (39\%), followed by beech (21\%), sycamore (11\%), rowan (11\%) and ash (9\%; Fig. 5). After adaptation thinning (variant B) the highest change was observed in decrease (by $5 \%$ ) of ash share due to weakening of trees by pests (see Study area). Share of birch decreased by $4 \%$, while share of sycamore increased by $3 \%$ and beech by $2 \%$. Share of all other tree species also increased. In variant $\mathrm{C}$ (thinning from below), share of

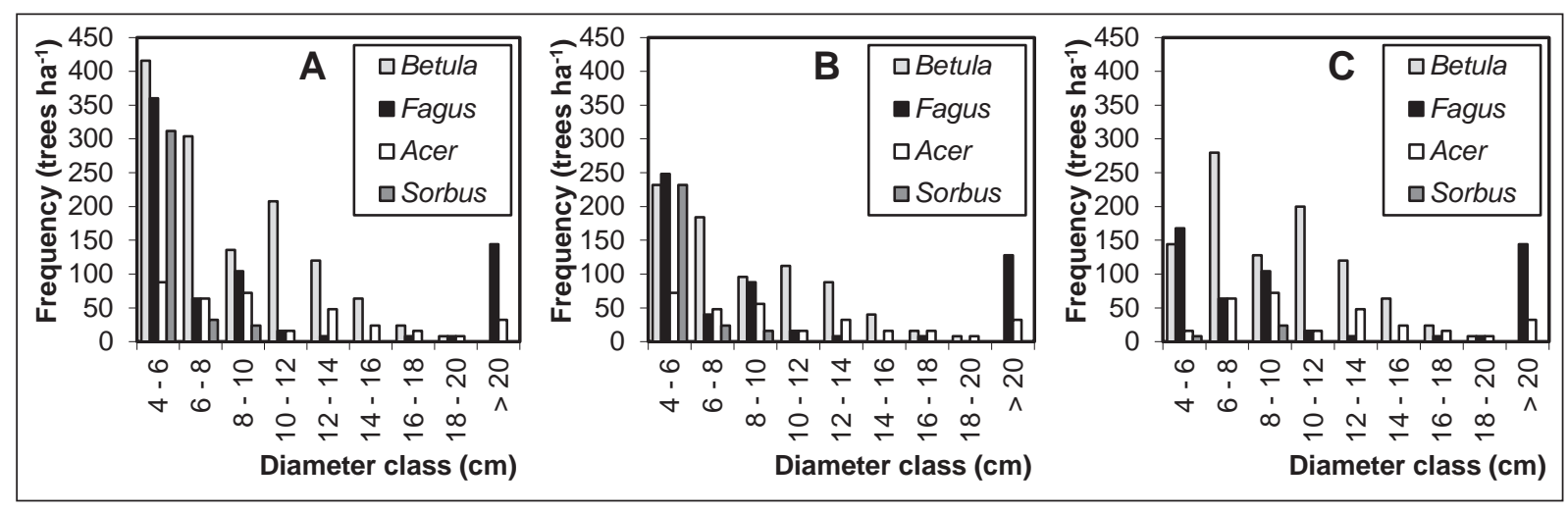

Fig. 3. Histogram of tree layer diameter structure according to main tree species in A) control variant (before thinning), B) after applied climate change adaptation thinning and C) after simulative thinning from below.

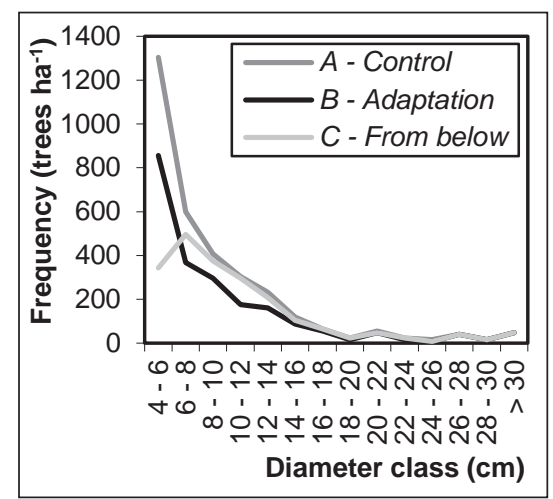

Fig. 4. Summary histogram of complete tree layer diameter structure in A) control variant (before thinning), B) after applied climate change adaptation thinning and C) after simulative thinning from below. rowan decreased by $10 \%$ and willow by $4 \%$ due to their occurrence predominantly in the understory. On the other hand, an increase was observed in beech (by 2\%) and especially in birch (by $8 \%$ ). Higher mean species richness $D$ was observed in variant $\mathrm{B}(0.573)$ and variant $\mathrm{A}$ (0.559) compared to variant $C(0.346$; Table 4$)$. Together with species heterogeneity $H^{\prime}$ both indices showed high diversity compared to moderate diversity in variant $C$. No differences were observed in species evenness $E$ among thinning variants indicating very high diversity (A-0.721, B - 0.726, C-0.736).

In terms of structural diversity, higher height differentiation $T M_{h}$ was observed in stands after adaptation thinning (0.292) and in controlvariant $(0.256)$ compared to thinning from below $(0.212$; Table 4$)$. Similarly, lower diameter differentiation $T M_{d}$ was observed in variant

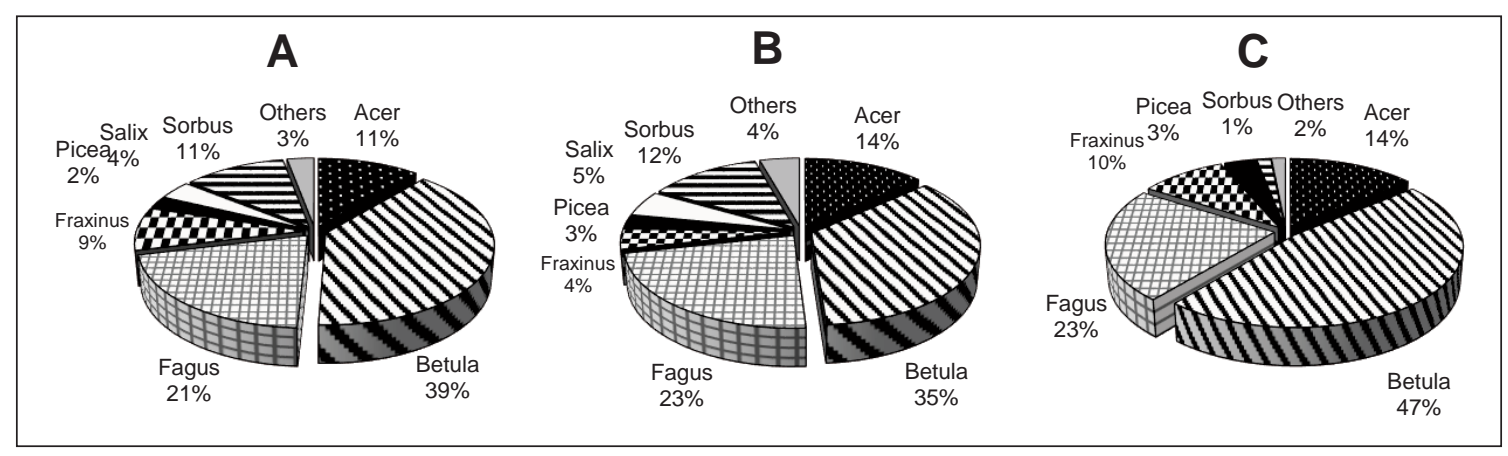

Fig. 5. Tree species composition in A) control variant (before thinning), B) after applied climate change adaptation thinning and C) after simulative thinning from below; others tree species include: Prunus avium, Abies alba and Populus tremula. 
Table 4. Tree layer biodiversity on experimental research plots 1-8 in A) control variant (before thinning), B) after applied climate change adaptation thinning and $\mathrm{C}$ ) after simulative thinning from below.

\begin{tabular}{|c|c|c|c|c|c|c|c|c|c|c|c|c|c|c|c|c|c|c|c|c|c|}
\hline ERP & Type & $\begin{array}{c}\mathrm{D} \\
{[\mathrm{Mi}]}\end{array}$ & & $\begin{array}{c}\mathrm{H}^{\prime} \\
{[\mathrm{Si}]}\end{array}$ & & $\begin{array}{c}\text { E } \\
{[\text { Pii }]}\end{array}$ & & $\begin{array}{c}\alpha^{*} \\
{[\mathrm{P} \& \mathrm{Mi}}\end{array}$ & & $\begin{array}{c}\mathrm{R}^{*} \\
{[\mathrm{C} \& \mathrm{Ei}]}\end{array}$ & & $\begin{array}{c}\mathrm{CS}^{*} \\
{[\mathrm{D} \& \mathrm{Mi}}\end{array}$ & & $\begin{array}{c}\text { A } \\
\text { [Pri] }\end{array}$ & & $\begin{array}{l}\mathrm{TM}_{\mathrm{d}} \\
{[\mathrm{Fi}]}\end{array}$ & & $\begin{array}{l}\mathrm{TM}_{\mathrm{h}} \\
\text { [Fi] }\end{array}$ & & $\begin{array}{c}\text { B } \\
{[\mathrm{J} \& \mathrm{Di}]}\end{array}$ & \\
\hline & $\mathrm{A}$ & 0.490 & & 0.576 & & 0.824 & & 1.602 & & 1.006 & & 0.189 & & 0.692 & & 0.371 & & 0.309 & & 7.846 & \\
\hline \multirow[t]{3}{*}{1} & B & 0.523 & $\nearrow$ & 0.540 & $\searrow$ & 0.773 & $\searrow$ & 1.303 & $\searrow$ & 1.141 & $\nearrow$ & -0.031 & $\searrow$ & 0.591 & $\searrow$ & 0.390 & $\nearrow$ & 0.287 & $\searrow$ & 7.596 & $\searrow$ \\
\hline & $\mathrm{C}$ & 0.385 & $\searrow$ & 0.560 & $\searrow$ & 0.930 & $\nearrow$ & 1.140 & $\searrow$ & 1.020 & $\nearrow$ & 0.116 & $\searrow$ & 0.719 & $\nearrow$ & 0.344 & $\searrow$ & 0.220 & $\searrow$ & 6.740 & $\searrow$ \\
\hline & $\mathrm{A}$ & 0.852 & & 0.797 & & 0.883 & & $1.677^{\mathrm{A}}$ & & $0.913^{\mathrm{A}}$ & & 0.086 & & 0.756 & & 0.308 & & 0.242 & & 9.111 & \\
\hline \multirow[t]{3}{*}{2} & B & 0.904 & $\nearrow$ & 0.802 & $\nearrow$ & 0.888 & $\nearrow$ & 1.568 & $\searrow$ & 0.979 & $\nearrow$ & -0.053 & $\searrow$ & 0.689 & $\searrow$ & 0.340 & $\nearrow$ & 0.277 & $\nearrow$ & 8.866 & $\searrow$ \\
\hline & $\mathrm{C}$ & 0.661 & $\searrow$ & 0.625 & $\searrow$ & 0.803 & $\searrow$ & 1.198 & $\searrow$ & 1.082 & $\nearrow$ & -0.088 & $\searrow$ & 0.717 & $\searrow$ & 0.306 & $\searrow$ & 0.225 & $\searrow$ & 7.840 & $\searrow$ \\
\hline & $\mathrm{A}$ & 0.721 & & 0.604 & & 0.715 & & $1.619^{\mathrm{A}}$ & & 0.980 & & 0.190 & & 0.672 & & 0.334 & & 0.244 & & 8.139 & \\
\hline \multirow[t]{3}{*}{3} & B & 0.631 & $\searrow$ & 0.566 & $\searrow$ & 0.727 & $\nearrow$ & 1.221 & $\searrow$ & 0.935 & $\searrow$ & -0.060 & $\searrow$ & 0.673 & $\nearrow$ & 0.354 & $\nearrow$ & 0.256 & $\nearrow$ & 7.422 & \\
\hline & $\mathrm{C}$ & 0.502 & $\searrow$ & 0.589 & $\searrow$ & 0.843 & $\nearrow$ & 1.561 & $\searrow$ & 0.987 & $\nearrow$ & -0.060 & $\searrow$ & 0.710 & $\nearrow$ & 0.306 & $\searrow$ & 0.205 & $\searrow$ & 6.887 & $\searrow$ \\
\hline & $\mathrm{A}$ & 0.772 & & 0.571 & & 0.676 & & 1.622 & & 1.009 & & 0.239 & & 0.664 & & 0.408 & & 0.310 & & 8.107 & \\
\hline \multirow[t]{3}{*}{4} & B & 0.805 & $\nearrow$ & 0.624 & $\nearrow$ & 0.738 & $\nearrow$ & 0.845 & 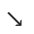 & 1.129 & $\nearrow$ & -0.085 & 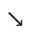 & 0.609 & $\searrow$ & 0.457 & $\nearrow$ & 0.367 & $\nearrow$ & 8.378 & $\lambda$ \\
\hline & $\mathrm{C}$ & 0.539 & $\searrow$ & 0.495 & $\searrow$ & 0.708 & $\nearrow$ & $2.073^{\mathrm{A}}$ & $\nearrow$ & $0.893^{\mathrm{A}}$ & $\searrow$ & 0.165 & $\searrow$ & 0.673 & $\nearrow$ & 0.396 & $\searrow$ & 0.249 & $\searrow$ & 7.105 & $\searrow$ \\
\hline & $\mathrm{A}$ & 0.254 & & 0.420 & & 0.880 & & 1.364 & & 1.085 & & 0.042 & & 0.793 & & 0.384 & & 0.259 & & 6.193 & \\
\hline \multirow[t]{3}{*}{5} & B & 0.270 & $\nearrow$ & 0.421 & $\nearrow$ & 0.894 & $\nearrow$ & 1.259 & $\searrow$ & 1.209 & $\nearrow$ & $-0.525^{\mathrm{R}}$ & $\searrow$ & 0.761 & $\searrow$ & 0.406 & $\nearrow$ & 0.261 & $\lambda$ & 5.866 & $\searrow$ \\
\hline & $\mathrm{C}$ & 0.133 & $\searrow$ & 0.301 & $\searrow$ & 1.000 & $\nearrow$ & 1.177 & $\searrow$ & 1.051 & $\searrow$ & -0.215 & $\searrow$ & 0.786 & $\nearrow$ & 0.344 & $\searrow$ & 0.229 & $\searrow$ & 4.356 & $\searrow$ \\
\hline & $\mathrm{A}$ & 0.351 & & 0.334 & & 0.555 & & $1.549^{\mathrm{A}}$ & & 0.962 & & 0.239 & & 0.551 & & 0.261 & & 0.197 & & 5.787 & \\
\hline \multirow[t]{3}{*}{6} & B & 0.362 & $\nearrow$ & 0.372 & $\nearrow$ & 0.618 & $\nearrow$ & 1.485 & $\searrow$ & 0.967 & $\nearrow$ & -0.185 & $\searrow$ & 0.558 & $\nearrow$ & 0.238 & $\searrow$ & 0.183 & $\searrow$ & 5.533 & $\searrow$ \\
\hline & $\mathrm{C}$ & 0.000 & $\searrow$ & 0.000 & $\searrow$ & 0.000 & $\searrow$ & 1.452 & $\searrow$ & 1.083 & $\nearrow$ & -0.210 & $\searrow$ & 0.495 & $\searrow$ & 0.210 & $\searrow$ & 0.117 & $\searrow$ & 3.310 & $\searrow$ \\
\hline & $\mathrm{A}$ & 0.513 & & 0.497 & & 0.711 & & 1.192 & & 1.133 & & -0.135 & & 0.519 & & 0.294 & & 0.233 & & 8.039 & \\
\hline \multirow[t]{3}{*}{7} & B & 0.537 & $\nearrow$ & 0.502 & $\nearrow$ & 0.714 & $\nearrow$ & 0.809 & $\searrow$ & $1.296^{\mathrm{R}}$ & $\nearrow$ & $-0.384^{\mathrm{R}}$ & $\searrow$ & 0.521 & $\nearrow$ & 0.390 & $\nearrow$ & 0.301 & $\nearrow$ & 8.044 & $\nearrow$ \\
\hline & $\mathrm{C}$ & 0.270 & $\searrow$ & 0.472 & $\searrow$ & 0.989 & $\nearrow$ & 1.535 & $\nearrow$ & 1.021 & $\searrow$ & -0.284 & $\searrow$ & 0.616 & $\nearrow$ & 0.276 & $\searrow$ & 0.197 & $\searrow$ & 5.945 & $\searrow$ \\
\hline & $\mathrm{A}$ & 0.523 & & 0.371 & & 0.531 & & $1.851^{\mathrm{A}}$ & & $0.663^{\mathrm{A}}$ & & -0.334 & & 0.510 & & 0.324 & & 0.267 & & 6.924 & \\
\hline \multirow[t]{2}{*}{8} & B & 0.559 & $\nearrow$ & 0.344 & $\searrow$ & 0.492 & $\searrow$ & 0.901 & $\searrow$ & 0.930 & $\nearrow$ & $-0.559^{R}$ & $\searrow$ & 0.459 & $\searrow$ & 0.501 & $\nearrow$ & 0.417 & $\nearrow$ & 6.492 & $\searrow$ \\
\hline & $\mathrm{C}$ & 0.276 & $\searrow$ & 0.295 & $\searrow$ & 0.618 & $\nearrow$ & 1.353 & $\searrow$ & 0.750 & $\nearrow$ & -0.348 & $\searrow$ & 0.546 & $\nearrow$ & 0.335 & $\nearrow$ & 0.254 & $\searrow$ & 6.039 & $\searrow$ \\
\hline
\end{tabular}

Notes: D - species richness, $\mathrm{H}^{\prime}$ - species heterogeneity, E - species evenness, $\alpha$-index of non-randomness, $\mathrm{R}$ - aggregation index, CS - index of cluster size, A-Arten-profile index, TM - index of diameter differentiation, $\mathrm{TM}_{\mathrm{h}}$ - index of height differentiation, $\mathrm{B}$ - stand diversity index; changes: $\downarrow$ - decrease, $>$ - increase;

* statistically significant $(\alpha=0.05)$ for horizontal structure $\left({ }^{\mathrm{A}}\right.$ - aggregation, ${ }^{\mathrm{R}}-$ regularity).

C (0.314) compared to variant B (0.383) and variant A (0.335). Structural differentiation was low to medium in all variants. No difference was found in vertical Artenprofile index $A(\mathrm{~A}-0.644, \mathrm{~B}-0.606, \mathrm{C}-0.657)$ that showed high diversity. Complex diversity index $B$ showed lower diversity in variant $C$ (6.027) compared to other two variants $(\mathrm{A}-7.518, \mathrm{~B}-7.257)$.

Differences were observed also in horizontal structure. Index of non-randomness showed higher tendency to aggregation in stands before intervention compared to thinning variants. Similarly, other both indices showed the highest tendency to regular spatial pattern of trees in variant $\mathrm{B}$. In the case of $5 \mathrm{ERP}$, horizontal structure moved from aggregated distribution to random, respectively from random to regular. Moreover, $L$-function showed shift from aggregated structure in variant A (from distance of $0.5 \mathrm{~m}$ ) to prevailing random structure in the other two variants, especially in adaptation thinning (Fig. 6).

\subsection{Interactions between stand parameters, structure, diversity and management}

The results of PCA are presented in an ordination diagram in Fig. 7. The first ordination axis explains $31.1 \%$ of data variability, the first two axes together explain $59.4 \%$ and the first four axes $84.7 \%$. The $\mathrm{x}$-axis illustrates the slenderness quotient (HDR) with crown closure (canopy) and the $y$-axis represents the mean stand height. Stand volume was positively correlated with mean diameter, tree volume and basal area, while these parameters were negatively correlated with tree density, HDR and index of non-randomness. Aggregation spatial pattern
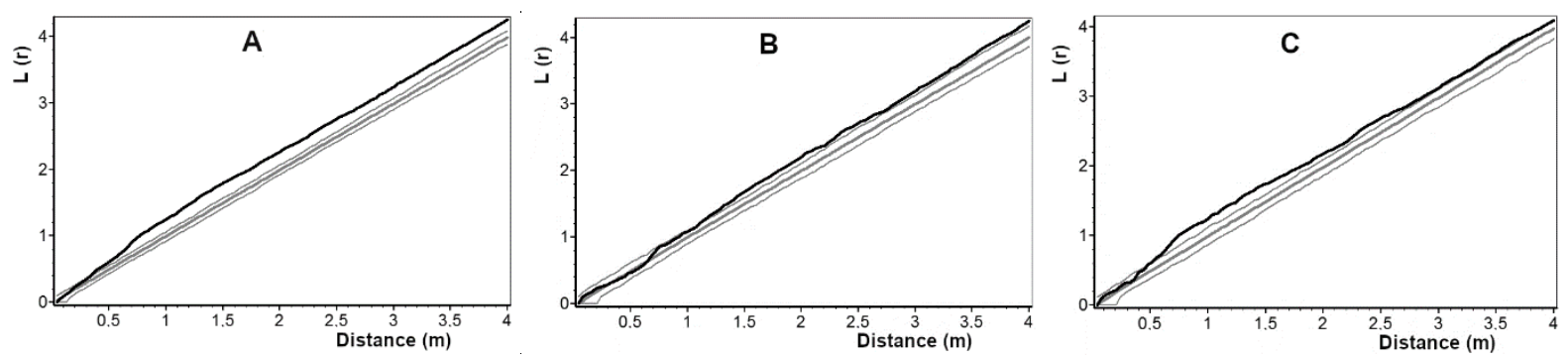

Fig. 6. Spatial pattern of tree layer on experimental research plots $1-8$ expressed by $L$-function in A) control variant (before thinning), B) after applied climate change adaptation thinning and C) after simulative thinning from below; the black line represents the $L$-function for real distances of trees; the bold gray line represents the mean course for random spatial distribution and the two thinner central curves represent $95 \%$ interval of reliability; when the black line of tree distribution on the ERP is under (below) this interval, it indicates a tendency of trees toward aggregation (regular) distribution; graphs made in software PointPro 2.1 (Zahradník \& Puš, ČZU). 
was increasing with increasing number of trees. Indices of diversity were positively correlated to each other. Mean height and species richness are the highest explanatory variable in ordination diagram compared to low explanation in SDI and Arten-profile index. Differences among all parameters were remarkable for ERP as marks of each records were relatively distant one from another whereas marks symbolizing variants of thinning were closer to each other, especially for adaptation thinning (variant B) and control variant A (before thinning). Before thinning, forest stands were characterized by high number of trees with aggregated structure, stands after applied adaptation thinning (variant B) had higher diameter and high differentiation and species evenness and the highest stand height was typical for simulative thinning from below (variant C). Generally, type of thinning had the highest effect (expected differences between plots) on mean height, species, structure and total diversity. strategy to cope with climate change (Jactel et al. 2009; Kolström et al. 2011; Bravo-Oviedo et al. 2014; Reims et al. 2015). Emphasis is at the same time placed on the variability of mixed forests corresponding to the given habitat and stand conditions (Seidl et al. 2011; Rio et al. 2016; Králíček et al. 2017; Vacek et al. 2017a; Mikulenka et al. 2020). Mixed forest tending is more complicated and require higher forestry expertise and qualification than tending of homogenous stands due to the need of consistently respecting the characteristics of tree species, inter- and intra-specific competition in close relation to habitat conditions (Slodičák \& Novák 2007; Poleno et al. 2009; Švec et al. 2015; Slanař et al. 2017).

Our results suggest that $33 \%$ of the trees, $22 \%$ of the basal area and $12 \%$ of the stand volume was harvested from the original number of 2,112 to 5,184 trees ha ${ }^{-1}$ in case of adaptation thinning, and it was $36 \%$ of the trees, $12 \%$ of the basal area and $6 \%$ of stand volume in case

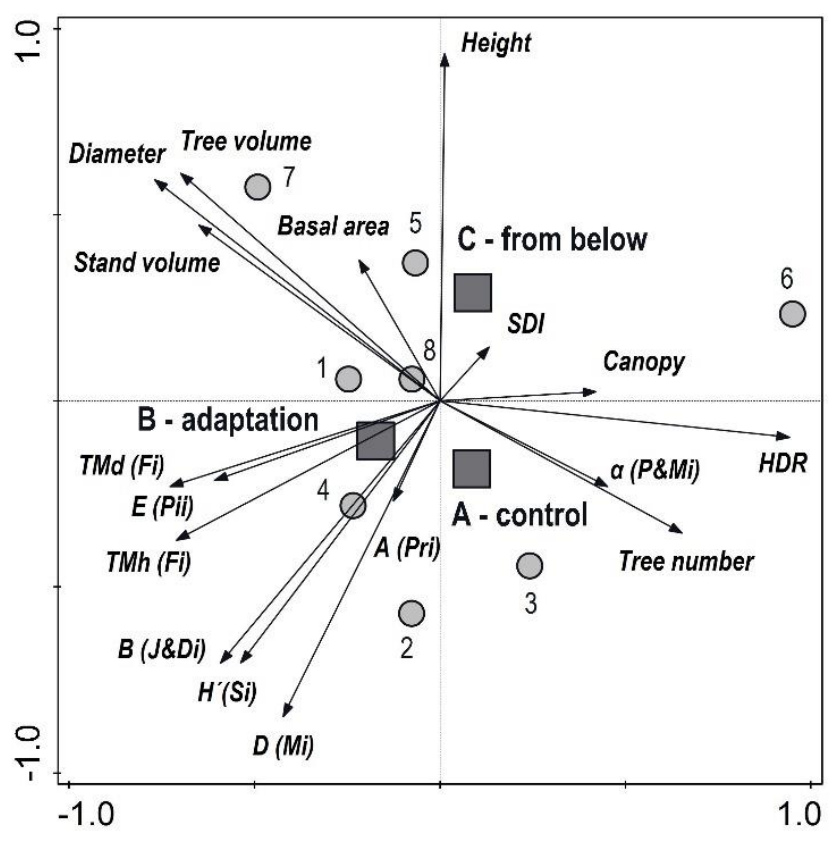

Fig. 7. Ordination diagram showing results of the PCA of relationships between variants of thinning (A - control before thinning, B - adaptation thinning, $\mathrm{C}$ - thinning from below), stand characteristics (Stand volume, Tree volume, Basal area, DBH, HDR, Height, Canopy, Tree number) and structural diversity $\left(D, H^{\prime}, E, A, T M d, T M h, \alpha\right.$ and $B$ indices); Symbols indicate plots 1-8 and variants of thinning (A, B, C).

\section{Discussion}

Climate change is currently one of the major challenges for the protection and management of forest ecosystems (Cotillas et al. 2009; Borys et al. 2016). It is therefore necessary to develop appropriate forestry strategies to mitigate the impact of global climate change on forest ecosystems (Chapin et al. 2001; Alvarez et al. 2016). In relation to ecological stability, it is then desirable to grow forests spatially, age and species differentiated (Brang et al. 2014). Promotion of mixed forests and species diversity is therefore often identified as a forest adaptation of thinning from below. For comparison, slightly higher density $\left(4,878-5,267\right.$ trees ha $\left.^{-1}\right)$ was found before the intervention in equally old (19years) mixed spruce-beech stands in South Bohemia (Novák \& Slodičák 2009). On the other hand, lower number of trees $(1,566-$ 2,240 trees ha $\left.{ }^{-1}\right)$, such as stand volume $\left(67-77 \mathrm{~m}^{3} \mathrm{ha}^{1}\right)$, was observed in mixed birch-dominated stands of age 20 years (Vacek et al. 2009). In parallel, there was an increase in average DBH by $8 \%$ and height by $4 \%$ for adaptation thinning and $16 \%$ in height and $\mathrm{DBH}$ for thinning from below. As in Waskiewicz et al. (2013), 
the aim of the stand tending was to improve the stability of the stand and keep the desired species composition in the young mixture. In our case, similarly to Novák et al. (2017), it is a lower intensity of the intervention, however, the internal stability of the stands of studied mixtures has been demonstrably increased. In connection with the intensity of the intervention, Konôpka et al. (2008) showed that even with higher intensity of the intervention ( $40 \%$ of the removed basal area), the stability of the stand can be significantly increased.

More intensive opening of forest stands has a greater impact on habitat and stand conditions, as evidenced by many researches (Poleno 1993, 1994; Jaworski 2000; Štefančík 2006, 2015; Rio et al. 2016). These are primarily microclimatic and ecological conditions, which can have a significant impact on the stability, production, health and vitality of stands (Chroust 1997). In the context of major global climate change, a number of papers from the Mediterranean conditions cite, for example, Roig et al. (2005); Gea-Izquierdo et al. (2009); Guillemot et al. (2015); Rio et al. (2016), that stronger tending interventions improve the moisture conditions in the stand and consequently the growth of the stand can be increased compared to stands without any intervention. Wherefore, higher opening of the vegetation under adaptation thinning according should have a more favourable effect on microclimatic and ecological conditions in the monitored stands compared to the thinning from below as well as on the diversity and development of herb layer in relation to light availability (Axmannová et al. 2011; Hédl et al. 2017). It is also noted that the high intensity of tending leads to an increase in the vitality of dominant trees in the stand after severe drought-stress events (Misson et al. 2003; Kohler et al. 2010; Sohn et al. 2013). Also, Bošel'a et al. (2016) showed that methods of differentiated tending of beech stands significantly influenced their vitality in conditions of the Carpathians, where the average temperature in the growing season has been rising since 1950.

The level of stress from water deficit is strongly influenced also by the species composition of stands. Prior to tending interventions, the main species on the experimental plots were birch (39\%), beech $(21 \%)$, sycamore (11\%), rowan (11\%) and ash (9\%). After adaptation thinning, the largest change in the species proportion was observed for ash as a result of damage by Hylesinus fraxini and Hymenoscyphus fraxineus, similarly to situation in other parts of the Krkonoše Mts. (Vacek et al. 2015 b , 2017b). Furthermore, the prevailing proportion of birch trees decreased, while there was a percentage increase in beech, sycamore, willow, rowan and other admixed tree species (cherry, aspen, fir). In case of thinning from below, the proportion of birch, beech and sycamore increased on the expense of the proportion of rowan and willow due to minimal economic use. In relation to tree species composition, researches showed a higher probability of drought stress in young spruce stands than in beech or other deciduous trees (Jelínek \& Kantor 2001; Borken \& Beese 2005; Novák \& Slodičák 2009). From this point of view, this proposed thinning could be a suitable adaptation option to decrease the risk of drought stress in forest stands.

In terms of diversity, adaptation thinning caused a shift in horizontal structure from aggregated to randomto-regular spatial distribution of trees. Furthermore, there was an increase in the diameter and height differentiation of the stand and an increase in species richness compared to the thinning from below or control variant. Thinning from below caused significant overall decrease in diversity of the stand. Our simulative results are consistent with Fahlvik et al. (2015), Holmstrom et al. (2016) and Novák et al. (2017), who show a significant influence of stand tending in young mixed stands on their structure. It should be noted that both the type of tending and the mixing, including the horizontal and vertical structure of the stands, modify the use of aboveground and underground resources and affect the distribution of growth among the trees in the stand and thereby the way of competition (Pretzsch \& Schütze 2014, 2015; Rio et al. 2014). More intensive tending intervention generally increases the growth capability of a stand and its vitality (Chroust 1997; Štefančík et al. 2018; Sharma et al. 2019). In case of adaptation thinning, we assumed that the new space created by tending intervention was necessary for the growth of promising future target trees, which will better resist the ongoing climate changes.

\section{Conclusion}

In this paper we tried to create a conceptual framework of the most important principles of stand tending, which take into an account the negative effects of global climate change on forest stands on the example of Krkonoše Mts. The proposed thinning system offers various ways of management, especially in terms of optimizing ecological and economic criteria for selecting tree species. The results show that adaptation thinning seems to be a more suitable option in given habitat and stand conditions compared to the thinning from below. There was an increase in structural diversity and species richness in adaptation thinning which is heading to higher stability of forest stands. In addition, it is important to ensure that the proposed silvicultural measures can deliver the required social and environmental functions and services of forest ecosystems continuously and in the highest possible quantity and quality. As a next step, different thinning approaches for different climate scenarios and site conditions should be developed and tested in field conditions, in order to verify their validity and feasibility. 


\section{Acknowledgments}

This study was supported by the Ministry of Agriculture of the Czech Republic (NAZVNo. QK1920328) and by the Czech University of Life Sciences Prague, Faculty of Forestry and Wood Sciences (IGA No. A02/19). We are grateful to two anonymous reviewers and editor for their constructive comments and valuable suggestions that helped improve the manuscript.

\section{References}

Aertsen, W., Janssen, E., Kint, V., Bontemps, J.-D., Van Orshoven, J., Muys, B., 2014: Long-term growth changes of common beech (Fagus sylvatica L.) are less pronounced on highly productive sites. Forest Ecology and Management, 312:252-259.

Agestam, E., Karlsson, M., Nilsson, U., 2006: Mixed forests as a part of sustainable forestry in southern Sweden. Journal of Sustainable Forestry, 21:101117.

Albert, M., Hansen, J., Nagel, J., Schmidt, M., Spellmann, H., 2015: Assessing risks and uncertainties in forest dynamics under different management scenarios and climate change. Forest Ecosystems, 2:7063.

Allen, C. D., Macalady, A. K., Chenchouni, H., Bachelet, D., McDowell, N., Vennetier, M. et al., 2010:Aglobal overview of drought and heat-induced tree mortality reveals emerging climate change risks for forests. Forest Ecology and Management, 259:660-684.

Alvarez, S., Ortiz, C., Díaz-Pinés, E., Rubio, A., 2016: Influence of tree species composition, thinning intensity and climate change on carbon sequestration in Mediterranean mountain forests: A case study using the $\mathrm{CO}_{2}$ Fix model. Mitigation and Adaptation Strategies for Global Change, 21:1045-1058.

Andivia, E., Rolo, V., Jonard, M., Formanek, P., Ponette, Q., 2016: Tree species identity mediates mechanisms of top soil carbon sequestration in a Norway spruce and European beech mixed forest. Annals of Forest Science, 73:437-447.

Axmanová, I., Chytrý, M., Zelený, D., Ching-Feng, L., Vymazalová, M., Danihelka, J. et al., 2011: The species richness-productivity relationship in the herb layer of European deciduous forests. Global Ecology and Biogeography, 21:657-667.

Bolte, A., Hilbrig, L., Grundmann, B., Kampf, F., Brunet, J., Roloff, A., 2010: Climate change impacts on stand structure and competitive interactions in a southern Swedish spruce-beech forest. European Journal of Forest Research, 129:261-276.

Borken, W., Beese, F., 2005: Soil carbon dioxide efflux in pure and mixed stands of oak and beech following removal of organic horizons. Canadian Journal of Forest Research, 35:2756-2764.

Borys, A., Suckow, F., Reyer, C., Gutsch, M., LaschBorn, P., 2016: The impact of climate change under different thinning regimes on carbon sequestration in a German forest district. Mitigation and Adaptation Strategies for Global Change, 21:861-881.
Bošela, M., Lukac, M., Castagneri, D., Sedmák, R., Biber, P., Carrer, M. et al., 2018: Contrasting effects of environmental change on the radial growth of co-occurring beech and fir trees across Europe. Science of the Total Environment, 615:1460-1469.

Bošela, M., Štefančík, I., Petráš, R., Vacek, S., 2016:The effects of climate warming on the growth of European beech forests depend critically on thinning strategy and site productivity. Agricultural and Forest Meteorology, 222:21-31.

Brang, P., Spathelf, P., Larsen, J.B., Bauhus, J., Bončina, A. et al., 2014: Suitability of close-to-nature silviculture for adapting temperate European forests to climate change. Forestry, 87:492-503.

Bravo-Oviedo, A., Pretzsch, H., Ammer, C., Andenmatten, E., Barbati, A., Barreiro, S. et al., 2014: European mixed forests: definition and research perspectives. Forest Systems, 23:518-533.

Bulušek, D., Vacek, Z., Vacek, S., Král, J., Bílek, L., Králíček, I., 2016: Spatial pattern of relict beech (Fagus sylvatica L.) forests in the Sudetes of the Czech Republic and Poland. Journal of Forest Science, 62:293-305.

Bussotti, F., Pollastrini, M., Holland, V., Bruggemann, W., 2015: Functional traits and adaptive capacity of European forests to climate change. Environmental and Experimental Botany, 111:91-113.

Chapin, F., Sala, O. E., Huber-Sannwald, E., 2001: Global biodiversity in a changing environment. Springer-Verlag, New York.

Chroust, L., 1997: Ekologie výchovy lesních porostů. Opočno, VÚLHM VS, 227 p.

Clark, P. J., Evans, F. C., 1954: Distance to nearest neighbour as a measure of spatial relationship in populations. Ecology, 35:445-453.

Coomes, D. A., Flores, O., Holdaway, R., Jucker, T., Lines, E. R., Vanderwel, M. C., 2014: Wood production response to climate change will depend critically on forest composition and structure. Global Change Biology, 20:3632-3645.

Cotillas, M., Sabate, S., Gracia, C., Espelta, J. M., 2009: Growth response of mixed mediterranean oak coppices to rainfall reduction Could selective thinning have any influence on it? Forest Ecology and Management, 258:1677-1683.

Cordonnier, T., Berger, F., Elkin, C., Lämås, T., Martinez., M., 2013: ARANGE Deliverable D2.2 Models and linker functions (indicators) for ecosystem services. $94 \mathrm{p}$.

Crookston, N. L., Stage, A. R., 1999: Percent canopy cover and stand structure statistics from the Forest Vegetation Simulator. Gen. Tech. Rep. RMRSGTR-24. Ogden, UT. U.S. Department of Agriculture, Forest Service, Rocky Mountain Research Station, $11 \mathrm{p}$.

Cukor, J., Vacek, Z., Linda, R., Bílek, L., 2017: Carbon sequestration in soil following afforestation of former agricultural land in the Czech Republic. Central European Forestry Journal, 63:97-104. 
David, F. N., Moore, P. G., 1954: Notes on contagious distribu-tions in plant populations. Annals of Botany of London, 18:47-53.

Dhar, A., Wang, J. R., Hawkins, C. D. B., 2015: Interaction of trembling aspen and lodgepole pine in a young sub-boreal mixedwood stand in central British Columbia. Open Journal of Forestry, 5:129-138.

Drössler, L., Övergaard, R., Ekö, P. M., Gemmel, P., Böhlenius, H., 2015: Early development of pure and mixed tree species plantations in Snogeholm, southern Sweden. Scandinavian Journal of Forest Research, 30:304-316.

Dyderski, M. K., Paź, S., Frelich, L. E., Jagodziński, A. M., 2017: How much does climate change threaten European forest tree species distributions? Global Change Biology, 24:1150-1163.

Fabrika, M., Ďurský, J., 2005: Stromové rastové simulátory. Zvolen, EFRA, 112 p.

Fahlvik, N.,Agestam, E., Nilsson, U., Nystrom, K., 2005: Simulating the influence of initial stand structure on the development of young mixtures of Norway spruce and birch. Forest Ecology and Management, 213:297-311.

Fahlvik, N., Ekö, P. M., Petersson, N., 2015: Effects of precommercial thinning strategies on stand structure and growth in a mixed even-aged stand of Scots pine, Norway spruce and birch in southern Sweden. Silva Fennica, 49:1-17.

Forrester, D.I., Pretzsch, H., 2015: Tamm review: On the strength of evidence when comparing ecosystem functions of mixtures with monocultures. Forest Ecology and Management, 356:41-53.

Füldner, K., 1995: Strukturbeschreibung in Mischbeständen. Forstarchiv, 66:235-606.

Gallo, J., Baláš, M., Linda, R., Kuneš, I., 2017: Growth performance and resistance to ground late frosts of Fagus sylvatica L. plantation treated with a brassinosteroid compound. Journal of Forest Science, 63:117-125.

Gallo, J., Bílek, L., Šimůnek, V., Roig, S., Fernández, J.A.B., 2020: Uneven-aged silviculture of Scots pine in Bohemia and Central Spain: comparison study of stand reaction to transition and long-term selection management. Journal of Forest Science, 66:22-35.

Gazol, A., Camarero, J. J., Gutierrez, E., Popa, I., AndreuHayles, L., Motta, R., Carrer, M., 2015: Distinct effects of climate warming on populations of silver fir (Abies alba) across Europe. Journal of Biogeography, 42:1150-1162.

Gea-Izquierdo, G., Martín-Benito, D., Cherubini, P., Isabel, C., 2009: Climate-growth variability in Quercus ilex L. west Iberian open woodlands of different stand density. Annals of Forest Science, 66:802.

Guillemot, J., Klein, E. K., Davi, H., Courbet, F., 2015: The effects of thinning intensity and tree size on the growth response to annual climate in Cedrus atlantica: a linear mixed modelingapproach. Annals of Forest Science, 72:651-663.
Hanewinkel, M., Cullmann, D. A., Schelhaas, M.-J., Nabuurs, G.-J., Zimmermann, N. E., 2013: Climate change may cause severe loss in the economic value of European forest land. Nature Climate Change, 3:203-207.

Hédl, R., Šipoš, J., Chudomelová M., Unitek, D., 2017: Dynamics of herbaceous vegetation during four years of experimental coppice introduction. Folia Geobotanica, 52:83-99.

Hember, R. A., Kurz, W. A., Metsaranta, J. M., Black, T. A., Guy, R. D., Coops, N. C., 2012: Accelerating regrowth of temperate-maritime forests due to environmental change. Global Change Biology, 18:20262040.

Holmstrom, E., Hjelm, K., Karlsson, M., Nilsson, U., 2016: Scenario analysis of planting density and precommercial thinning: Will the mixed forest have a chance? European Journal of Forest Research, 135:885-895.

Hynynen, J., Repola, J., Mielikäinen, K., 2011:The effects of species mixture on the growth and yield of mid-rotation mixed stands of Scots pine and silver birch. Forest Ecology and Management, 262:1174-1183.

Jactel, H., Nicoll, B. C., Branco, M., Gonzalez-Olabarria, J. R., Grodzki, W, Långström, B. et al., 2009: The influences of foreststand management on biotic and abiotic risks of damage. Annals of Forest Science, 66:701.

Jaehne, S. C., Dohrenbusch, A., 1997: Ein Verfahren zur Beurteilung der Bestandesdiversität. Forstwissenschaftliches Centralblatt, 116:333-345.

Jaworski, A., 2000: Zasady hodowli lasów górských na podstawach ekologicznych. Warszawa.

Jelínek, P., Kantor, P., 2001: Production potential and ecological stability of mixed forest stands in uplandsIV.A mixed spruce/pine stand in the forest type group 2S (fresh, nutrient-medium beech-oak stand). Journal of Forest Science, 47:529-544.

Jönsson, A. M., Appelberg, G., Harding, S., Bärring, L., 2009: Spatio-temporal impact of climate change on the activity and voltinism of the spruce bark beetle, Ips typographus. Global Change Biology, 15:486-499.

Knoke, T., Ammer, C., Stimm, B., Mosandl, R., 2008: Admixing broadleaved to coniferous tree species: a review on yield, ecological stability and economics. European Journal of Forest Research, 127:89-101.

Kohler, M., Sohn, J., Nägele, G., Bauhus, J., 2010: Can drought tolerance of Norway spruce (Picea abies [L.] Karst.) be increased through thinning? European Journal of Forest Research, 129:1109-1118.

Kolström, M., Lindner, M., Vilén, T., Maroschek, M., Seidl, R., Lexer, M. J. et al., 2011: Reviewing the science and implementation of climate change adaptation measures in European forestry. Forests, 2:961-982.

Konôpka, J., Konôpka, B., Nikolov, C., 2008: Analysis of salvage timber felling according to injurious agents and forest regions. Lesnícky časopis - Forestry Journal, 54:107-126. 
Köppen, W., 1931: Grundriss der Klimakunde. (Zweite, verbesserte Auflage der Klimate der Erde.). Walter de Gruyter \& Co., Berlin.

Kozak, I., Parpan, T., 2019: Forecasting drying up of spruce forests in Transcarpathia (Ukraine) using the FORKOME model. Journal of Forest Science, 65:209-217.

Kraft, G., 1884: Beiträgezur zur lehre von den durchforstungen, schlagstellungen und lichtungshieben. Klindworth, Hannover, Germany.

Králíček, I., Vacek, Z., Vacek, S., Remeš, J., Bulušek, D., Král, J. et al., 2017: Dynamics and structure of mountain autochthonous spruce-beech forests: impact of hilltop phenomenon, air pollutants and climate. Dendrobiology, 77:119-137.

Liang, J., Crowther, T. W., Picard, N., Wiser, S., Zhou, M., Alberti, G. et al., 2016: Positive biodiversity-productivity relationship predominant in global forests. Science, 354(6309): aaf8957.

Lindner, M., Fitzgerald, J. B., Zimmermann, N. E., Reyer, Ch., Delzon, S., der Maaten, E. et al., 2014: Climate change and European forests: What do we know, what are the uncertainties, and what are the implications for forest management? Journal of Environmental Management, 146:69-83.

Lindner, M., Maroschek, M., Netherer, S., Kremer, A., Barbati, A., Jordi Garcia-Gonzalo, J. et al., 2010: Climate change impacts, adaptive capacity, and vulnerability of European forest ecosystems. Forest Ecology and Management, 259:698-709.

Margalef, R., 1958: Information theory in ecology. General Systematics, 3:36-71.

Metz, J., Annighofer, P., Schall, P., Zimmermann, J., Kahl, T., Schulze, E.D., Ammer, C., 2016: Site adapted admixed tree species reduce drought susceptibility of mature European beech. Global Change Biology, 22:903-920.

Mikulenka, P., Prokůpková, A., Vacek, Z., Vacek, S., Bulušek, D., Simon, J. et al., 2020: Effect of climate and air pollution on radial growth of mixed forests: Abies alba (Mill.) vs. Picea abies (L.) Karst. Central European Forestry Journal, 66:23-36.

Misson, L., Vincke, C., Devillez, F., 2003: Frequency responses of radial growth series after different thinning intensities in Norway spruce (Picea abies [L.] Karst.) stands. Forest Ecology and Management, 177:51-63.

Moreno-Fernández, D., Hernández, L., SánchezGonzález, M., Cañellas, I., Montes, F., 2016: Space-time modeling of changes in the abundance and distribution of tree species. Forest Ecology and Management, 372:206-216.

Mountford, M. D., 1961: On E. C. Pielou's index of nonrandomness. Journal of Ecology, 49:271-275.

Neuner, S., Beinhofer, B., Knoke, T., 2013: The optimal tree species composition for a private forest enterprise-applying the theory of portfolio selection. Scandinavian Journal of Forest Research, 28:38-48.
Novák, J., Dušek, D., Slodičák, M., Kacálek, D., 2017: Importance of the first thinning in young mixed Norway spruce and European beech stands. Journal of Forest Science, 63:254-262.

Novák, J., Slodičák, M., 2009: Thinning experiment in the spruce and beech mixed stands on the locality naturally dominated by beech - growth, litter-fall and humus. Journal of Forest Science, 55:224-233.

O’Hara, K. L., 2015: What is close-to-nature silviculture in a Changing world? Forestry, 89:1-6.

O’Connor, M. I., Holding, J. M., Kappel, C. V., Duarte, C. M., Brander, K., Brown, C. J. et al., 2015: Strengthening confidence in climate change impact science. Global Ecology and Biogeography, 24:64-76.

Petráš, R., Pajtík, J., 1991: Sústava česko-slovenských objemových tabuliek drevín. Lesnícky časopis, 37:49-56.

Pielou, E. C., 1959: The use of point-to-plant distances in the study of the pattern of plant populations. Journal of Ecology, 47:607-613.

Pielou, E. C., 1975: Ecological diversity. New York: Wiley, USA, $165 \mathrm{p}$.

Piovesan, G., Biondi, F., Di Filippo, A., Alessandrini, A., Maugeri, M., 2008: Drought-driven growth reduction in old beech (Fagus sylvatica L.) forests of the central Apennines, Italy. Global Change Biology, 14:1265-1281.

Podrázský, V., Zahradník, D., Remeš, J., 2014: Potential consequences of tree species and age structure changes of forests in the Czech Republic - review of forest inventory data. Wood Research, 59:483-490.

Podrázský, V., 2019: State of beech pole stands established at the clear-cut and in the underplanting. Journal of Forest Science, 65:256-262.

Pokorný, R., 2013: Pěstování lesů pod vlivem měnícího se klimatu. Brno, Lesnická a dřevařská fakulta, Mendelova univerzita v Brně, 39 p.

Poleno, Z., 1993: Ekologicky orientované pěstování lesů I. Lesnictví - Forestry, 39:475-480.

Poleno, Z., 1994: Ekologicky orientované pěstovánílesů II. Lesnictví - Forestry, 40:65-72.

Poleno, Z., Vacek, S., Podrázský, V., Remeš, J., Štefančík, I., Mikeska, M. et al., 2009: Pěstování lesů III. Praktické postupy pěstování lesů. Kostelec nad Černými lesy, Lesnická práce, 952 p.

Pretzsch, H., 2006: Wissen nutzbar machen für das Management von Waldökosystemen. Allgemeine Forstzeitschrift/Der Wald, 61:1158-1159.

Pretzsch, H., Biber, P., Schütze, G., Uhl, E., Rötzer, T., 2014: Forest stand growthdynamics in Central Europe haveacceleratedsince 1870. Nature Communications, 5:4967.

Pretzsch, H., Schütze, G., 2014: Size-structure dynamics of mixed versus pure forest stands. Forest Systems, 23:560-572.

Pretzsch, H., Schütze, G., 2015: Effect of tree species mixing on the sizestructure, density and yield forest stands. European Journal of Forest Research, 135:1-22. 
Pretzsch, H., Biber, P., 2016: Tree species mixing can increase maximum stand density. Canadian Journal of Forest Research, 46:1179-1193.

Prévost, M., Gauthier, M.-M., 2012: Precommercial thinning increases growth of overstory aspen and understory balsam fir in a boreal mixedwood stand. Forest Ecology and Management, 278:17-26.

Primicia, I., Camarero, J. J., Janda, P., Čada, V., Morrissey, R. C., Trotsiuk, V. et al., 2015:Age, competition, disturbance and elevation effects on tree and stand growth response of primary Picea abies forest to climate. Forest Ecology and Management, 354:77-86.

Pukkala, T., 2018: Effect of species composition on ecosystem services in European boreal forest. Journal of Forest Research, 29:261-272.

Quitt, E., 1971: Klimatické oblasti Československa. Academia, Praha, 73 p.

Rabasa, S. G., Granda, E., Benavides, R., Kunstler, G., Espelta, J. M., Ogaya, R. et al., 2013: Disparity in elevational shifts of European trees in response to recent climate warming. Global Change Biology, 19:2490-2499.

Reineke, L. H., 1933: Perfecting a stand-density index for even-aged forests. Journal of Agricultural Research, 46:627-638.

Remeš, J., Bílek, L., Novák, J., Vacek, Z., Vacek, S., Putalová, T., Koubek, L., 2015: Diameter increment of beech in relation to social position of trees, climate characteristics and thinning intensity. Journal of Forest Science, 61:456-464.

Rigling, A., Bigler, C., Eilmann, B., Feldmeyer-Christe, E., Gimmi, U., Ginzler, C. et al., 2013: Driving factors of a vegetation shift from Scots pine to pubescent oakin dry Alpine forests. Global Change Biology, 19:229-240.

Río, M., Pretzsch, H., Alberdi, I., Bielak, K., Bravo, F., Brunner, A. et al., 2016: Characterization of the structure, dynamics, and productivity of mixed-species stands: review and perspectives. European Journal of Forest Research, 135:23-49.

Ripley, B. D., 1981: Spatial Statistics. New York, John Wiley \& Sons: 252.

Roig, S., Rio, M., Cañellas, I., Montero, G., 2005: Litter fall in Mediterranean Pinus pinaster Ait. stands under different thinning regimes. Forest Ecology and Management, 206:179-190.

Saltré, F., Duputié, A., Gaucherel, C., Chuine, I., 2014: How climate, migration ability and habitat fragmentation affect the projected future distribution of European beech. Global Change Biology, 21:897-910.

Scheffers, B. R., Meester, L. D., Bridge, T. C. L., Hoffmann, A. A., Pandolfi, J. M., Corlett, R. T., Watson, J. E. M., 2016: The broad footprint of climate change from genes to biomes to people. Science, 354:aaf7671.

Seidl, R., Aggestam, F., Rammer, W., Blennow, K., Wolfslehner, B., 2016: The sensitivity of current and future forest managers to climate-induced changes in ecological processes. Ambio, 45:430-441.
Seidl, R., Rammer, W., Lexar, M., 2011: Climate change vulnerability of sustainable forest management in the Eastern Alps. Climatic Change, 106:225-254.

Seidl, R., Schelhaas, M.-J., Rammer, W., Verkerk, P. J., 2014: Increasing forest disturbances in Europe and their impact on carbon storage. Nature Climate Change, 4:806-810.

Shannon, C. E., 1948: A mathematical theory of communications. Bell System Technical Journal, 27:379423.

Sharma, R. P., Štefančík, I., Vacek, Z., Vacek, S., 2019: Generalized Nonlinear Mixed-Effects Individual Tree Diameter Increment Models for Beech Forests in Slovakia. Forests, 10:451.

Slanař, J., Vacek, Z., Vacek, S., Bulušek, D., Cukor, J., Štefančík, I. et al., 2017: Long-term transformation of submontane spruce-beech forests in the Jizerské hory Mts.: dynamics of natural regeneration. Central European Forestry Journal, 63:213-225.

Slodičák, M., Novák, J., 2007: Výchova lesních porostů hlavních hospodářských dřevin. Lesnický průvodce, č. 4, Júloviště - Strnady, VÚLHM, 46 p.

Šmilauer, P., Lepš, J., 2014: Multivariate analysis of ecological data using CANOCO 5. Cambridge university press, $361 \mathrm{p}$.

Šimůnek, V., Vacek, Z., Vacek, S., Králíček, I., Vančura, K., 2019: Growth variability of European beech (Fagus sylvatica L.) natural forests: Dendroclimatic study from Krkonoše National Park. Central European Forestry Journal, 65:92-102.

Švec, O., Bílek, L., Remeš, J., Vacek, Z., 2015: Analysis of operational approach during forest transformation in Klokočná Range, Central Bohemia. Journal of Forest Science, 61:148-155.

Sohn, J.A., Gebhardt, T., Ammer, C., Bauhus, J., Häberle, K. H., Matyssek, R., Grams, T. E., 2013: Mitigation of drought by thinning: short-term and long-term effects on growth and physiological performance of Norway spruce (Picea abies). Forest Ecology and Management 308:188-197.

Spathelf, P., van der Maaten, E., van der Maaten-Theunissen, M., Campioli, M., Dobrowolska, D., 2014: Climate change impacts in European forests: the expert views of local observers. Annals of Forest Science, 71:131-137.

Štefančík, I., 2006: Changes in tree species composition, stand structure, qualitative and quantitative production of mixed spruce, fir and beech stand on Stará Píla research plot. Journal of Forest Science, 52:74-91.

Štefančík, I., Kamenský, M., 2006: Natural change of tree species composition in mixed spruce, fir and beech stands under conditions of climate change. Lesnícky časopis - Forestry Journal, 52:61-73.

Štefančík, I., 2010: Vplyv výchovy a poškodenia snehom na zmeny drevinového zloženia, porastovej výstavby, kvalitatívnej a kvantitatívnej produkcie zmiešanej smrekovojedlovo-bukovej žrd'oviny na výskumnej ploche Stará píla. Lesnícky časopis - Forestry Journal, 56:129-154. 
Štefančík, I., 2015: Rast, štruktúra a produkc ia bukových porastov s rozdielnym režimom výchovy. Zvolen, NLC, $148 \mathrm{p}$.

Štefančík, I., Vacek, S., Podrázský, V., 2018: The most significant results of long-term research on silviculture experiments focusing on spruce and beech in the territory of the former Czechoslovakia. Central European Forestry Journal, 64:180-194.

Štefančík, I., Vacek, Z., Sharma, R. P., Vacek, S., Rösslová, M., 2018: Effect of thinning regimes on growth and development of crop trees in Fagus sylvatica stands of Central Europe over fifty years. Dendrobiology, 79:141-155.

Vacek, S., Simon, J. et al., 2009: Zakládání a stabilizace lesních porostů na bývalých zemědělských a degradovaných půdách. Kostelec nad Černými lesy, Lesnická práce, $792 \mathrm{p}$.

Vacek, S., Hejcman, M., 2012: Natural layering, foliation, fertility and plant species composition of a Fagus sylvatica stand above the alpine timberline in the Giant (Krkonoše) Mts., Czech Republic. European Journal of Forest Research, 131:799-810.

Vacek, S., Vacek, Z., Podrazský, V., Bílek, L., Bulušek, D., Štefančík, I., 2014: Structural Diversity of Autochthonous Beech Forests in Broumovske Stěny National Nature Reserve, Czech Republic. Austrian Journal of Forest Science, 131:191-214.

Vacek, S., Vacek, Z., Bulušek, D., Bílek, L., Schwarz, O., Simon, J., Štícha, V., 2015a: The role of shelterwood cutting and protection against game browsing for the regeneration of silver fir. Austrian Journal of Forest Science, 132:81-102.

Vacek, S., Vacek, Z., Bulušek, D., Putalová, T., Sarginci, M., Schwarz, O. et al., 2015b: European Ash (Fraxinus excelsior $\mathrm{L}$.) Dieback: Disintegrating forest in the mountain protected areas, Czech Republic. Austrian Journal of Forest Science, 4:203-223.

Vacek, S., Černý, T., Vacek, Z., Podrázský, V., Mikeska, M., Králičck, I., 2017a: Long-term changes in vegetation and site conditions in beech and spruce forests of lower mountain ranges of Central Europe. Forest Ecology and Management, 398:75-90.
Vacek, S., Prokůpková, A., Vacek, Z., Bulušek, D., Šimůnek, V., Králíček, I. et al., 2019b: Growth response of mixed beech forests to climate change, various management and game pressure in Central Europe. Journal of Forest Science, 65:331-345.

Vacek, Z., Vacek, S., Bulušek, D., Podrázský, V., Remeš, J., Král, J., Putalová, T., 2017b: Effect of fungal pathogens and climatic factors on production, biodiversity and health status of ash mountain forests. Dendrobiology, 77:1755-1315.

Vacek, Z., Vacek, S., Slanař, J., Bílek, L., Bulušek, D., Štefančík, I. et al., 2019a:Adaption of Norway spruce and European beech forests under climate change: from resistance to close-to-nature silviculture. Central European Forestry Journal, 65:129-144.

Viewegh, J., Kusbach, A., Mikeska, M., 2003: Czech forest ecosystem classification. Journal of Forest Science, 49:74-82.

Vinš, B. et al., 1997: Impacts of a Potential Climate Change on Forests of the Czech Republic. Národní klimatický program, 23. ČHMÚ, 142 p.

Vitali, V., Forrester, D. I., Bauhus, J., 2018: Know your neighbours: drought response of Norway Spruce, Silver Fir and Douglas Fir in mixed forests depends on species identity and diversity of tree neighbourhoods. Ecosystems, 21:1215-1229.

Waskiewicz, J., Kenefic, L., Weiskittel, A., Seymour, R., 2013: Species mixture effects in northern red oak-eastern white pine stands in Maine, USA. Forest Ecology and Management, 298:71-81.

Zimmermann, J., Hauck, M., Dulamsuren, C., Leuschner, C., 2015: Climate Warming-Related Growth Decline Affects Fagus sylvatica, But Not Other Broad-Leaved Tree Species in Central European Mixed Forests. Ecosystems, 1:560-572.

\section{Other sources}

Forest Europe, 2015: Madrid Ministerial Declaration: 25 years together promoting Sustainable Forest Management in Europe. 7th Conference Ministerial Forest Europe, Madrid.

IPCC, 2013: Climate change 2013: The physical science basis. Contribution of working group I to the fifth assessment report of the intergovernmental panel on climate change. Cambridge, New York: Cambridge University Press. 